\title{
Prevalence and Parasite Density of Asymptomatic Malaria Parasitemia among Unbooked Paturients at Abakaliki, Nigeria
}

\author{
Nwali Matthew Igwe, Umeora Odidika Ugochukwu Joannes, Ozumba Benjamin Chukwuma ${ }^{1}$, Onoh Robinson Chukwudi, \\ Ezeonu Paul Oliaemeka, Agwu Uzoma Maryrose, Agboeze Joseph \\ Federal Teaching Hospital, Abakaliki, Ebonyi, Nigeria, 'University of Nigeria Teaching Hospital, Ituku-Ozalla, Enugu, Nigeria
}

\section{A B S T R A C T}

Background: Malaria in pregnancy has contributed significantly to maternal morbidity and mortality in our environment. Aim: This study was aimed at determining the prevalence, and parasite density of asymptomatic malaria parasitemia among unbooked paturients at Federal Teaching Hospital Abakaliki. Subjects and Methods: This was a prospective cross-sectional study conducted in the labor ward complex of the Federal Teaching Hospital Abakaliki over a period of three months from March to May 2012. Two hundred and fifty unbooked women presenting in labor at term and willing to participate were consecutively recruited. A structured data collection sheet was administered to each parturient. Thick and thin blood films were prepared for quantification and speciation of parasitemia respectively. Results: A total of 250 unbooked parturients participated in the study. The mean age of the parturients was 28.2 (7.2) years. One hundred and seven $107 / 250$ (42.8\%) were primigravid, 111/250 (44.4\%) were between para 1-4 while 32/250 (12.8\%) were para 5 and above. Majority of the parturients 236/250 (95.2\%) were of low socio-economic class. The prevalence of asymptomatic malaria parasitemia was $77.6 \%$. Asymptomatic malaria parasitemia was most prevalent among paturients of social class $5(24 / 32(75 \%)$ and primigravid paturients (93/107 (86.9\%)) and the association of asymptomatic malaria parasitemia with parity $\left(X^{2}=10.8, P=0.01\right)$ and social class $\left(X^{2}=10.88, P=0.02\right)$ was statistically significant. Conclusion: There was high prevalence of asymptomatic malaria parasitemia in the study. Preconceptional care and early antenatal booking are advocated with emphasis on prevention of malaria infestation through health education and effective use of insecticide treated nets and intermittent prophylaxis therapy for malaria during pregnancy.

KEY WORDS: Asymptomatic malaria parasitemia, Nigeria, prevalence, unbooked paturients

\section{INTRODUCTION}

Malaria is a parasitic disease of humans especially in the sub-Saharan Africa, where about 90\% of deaths due to malaria occur..$^{[1]}$ The term malaria originated from the medieval Italian; "mala aria" which means "bad air" and the disease was formerly called ague or marsh fever due to its association with swamp and marsh land. ${ }^{[2]}$ Malaria is a vector borne disease caused by the parasite of the genus Plasmodium. It is transmitted by the bite of an infected female anopheles mosquito. Four species are mainly responsible for human malaria infestation, Plasmodium falciparum, Plasmodium malariae, Plasmodium ovale, and Plasmodium vivax. Plasmodium falciparum is the commonest in Africa and is responsible for up to $98 \%$ of cases in Nigeria

\begin{tabular}{|l|l|}
\hline \multicolumn{2}{|c|}{ Access this article online } \\
\hline Quick Response Code & Website: \\
& www.jbcrs.org \\
\cline { 2 - 2 } & DOI: \\
& $* * *$ \\
\hline
\end{tabular}

and is associated with severe morbidity and mortality. ${ }^{[3,4]}$ Plasmodium malariae and ovale are responsible for $2 \%$ of cases while Plasmodium vivax is not found among indigenous Nigerians. ${ }^{[4]}$

Pregnant women and children are especially susceptible to malaria in endemic regions due to low immunity. ${ }^{[1]}$ The burden of the disease is enormous in malaria-endemic countries like Nigeria. Each year, approximately 50 million women living in malaria-endemic areas throughout the world become pregnant, of which more than half live in tropical Africa with intense transmission of $P$. falciparum..$^{[5]}$ An estimate of 10,000 of these women and 200,000 of their infants die as a result of malaria. ${ }^{[5]}$ In Nigeria, malaria accounts for $60 \%$ of outpatient consultations and $11 \%$ of maternal mortality is due to malaria in pregnancy ${ }^{[3,4]}$ Malaria in pregnancy is an important cause of anemia, miscarriages, intrauterine growth restriction, low birth weight, still birth,

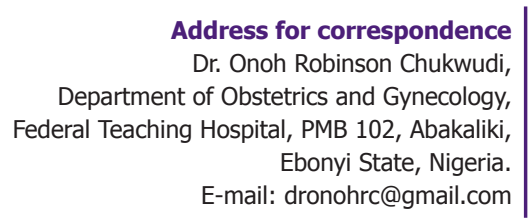


and other pregnancy-related complications. ${ }^{[3]}$ Seventy percent of pregnant women in Nigeria suffer malaria with maternal and fetal complications. ${ }^{[3]}$ The problem is compounded by high level of resistance to first- and second-line antimalariae drugs as shown by the drug therapeutic efficacy trial conducted in the six geopolitical zones of the country which showed resistance ranging from $23 \%$ to $96 \%{ }^{[4]}$

Malaria presents with different symptoms and signs. The common symptoms are intermittent fever, body aches and pains, malaise, generalized body weakness, etc., It is termed asymptomatic when there is malaria parasitemia in the absence of these symptoms. Most pregnant women with malaria parasitemia are asymptomatic. ${ }^{[1]}$ Up to $16-95.4 \%$ of asymptomatic parasitemia in pregnancy had been reported in various studies with associated maternal anemia and low birth weight..$^{[6-13]}$

This study was aimed at determining the prevalence of asymptomatic malaria parasitemia among unbooked clients presenting in labor in the Federal Teaching Hospital Abakaliki. This will help to detect those who have the problem and appropriate treatment administered in the short run and to show the enormity of the problem to help create awareness and to scale up preventive measures against this public health problem in the long run.

\section{SUBJECTS AND METHODS}

This was a prospective cross-sectional study conducted in the labor ward complex of the Federal Teaching Hospital, Abakaliki, Ebonyi state, Nigeria over a 3 month period from March to May 2012. The state is made up of 13 local government areas, one urban, one semiurban, and some rural areas. It occupies a land mass of $5932 \mathrm{~km}^{2}$ and has the population of 2.1 million people as was reported in 2006 by the National Population Commission. About $75 \%$ of the population are rural dwellers with subsistence farming as their major occupation. ${ }^{[14]}$ Malaria transmission is endemic in the state. The Federal Teaching Hospital is located in Abakaliki the capital city of the state. This is the major tertiary hospital in the state.

The study population included all unbooked women who presented in labor at term within the study period. The exclusion criteria were those who refused consent, symptomatic infection (fever, chills, rigor, nausea, vomiting, headache), preterm labor, and HIV in pregnancy. A structured data sheet was administered to the women by the authors after explaining the purpose of the study and obtaining informed consent which was later reconfirmed in the postnatal ward after delivery as participants are allowed to opt out of the study if they so desire in the course of the study. Also women in labor could give consent as a result of their condition and the painful distress of labor; hence, there is a need to reconfirm the consent in the postnatal ward. Information obtained included age, parity, gestational age, and social class.

Peripheral blood was collected from the antecubital vein via an aseptic procedure and the blood sample was used to prepare two thick and two thin films on prelabeled glass slides for each parturient. The thick film was for identification of malaria parasite, while the thin film was for identification of Plasmodium species. The thick blood films were allowed to air dry at room temperature after which they were stained with $5 \%$ Giemsa stain for 20 minutes. Thereafter they were rinsed with water, were air dried, and underwent microscopic examination under oil immersion at $100 \times$ magnification. The presence of $1-10$ parasites $/ 100$ thick film fields $(0.25 \mu \mathrm{L}$ of blood) or more was regarded as positive. The thin blood films were allowed to air dry and were then fixed with absolute methanol for 1 minute after which they were stained with Giemsa stain for 20 minutes, then rinsed with water and allowed to air dry before microscopic examination was done to identify the Plasmodium species. The samples were prepared, read, and analyzed by each of two experienced laboratory scientists dedicated to the study to ensure quality control. We employed the World Health Organization (WHO) semiquantitative method of parasite density estimation which is done by visualizing a 100 -field perimeter which is equivalent to $0.25 \mu \mathrm{L}$ of blood which is the method adopted in our hospital. The research and ethics committee of the Federal Teaching Hospital approved the study protocol. Those diagnosed with asymptomatic parasitemia were managed according to the Obstetrics and Gynecology departmental protocol. Asymptomatic malaria parasitemia is the identification of malaria parasite in the blood film of a parturient who had no symptoms of malaria infestation.

The minimum sample size of 206 women for the study was computed using $16^{[13]}$ prevalence from a similar study in the state using the formula proposed by Daniel, Lwanga, and Lameshow. ${ }^{[15,16]}$ This was increased to 247 women to control for attrition. The coded data was fed into the computer using Epi info program (2005) of CDC Atlanta USA 3.3.2 version and analysis done. A $P$ value less than 0.05 was considered statistically significant.

\section{RESULTS}

A total of 250 unbooked parturients in labor at term were recruited into the study out of which 194/250 (77.6\%) had asymptomatic malaria parasitemia. The mean age of the participants was 28.2 (7.2) years and ranged between 
19-45 years. The median parity was 2 (1.7) and ranged between para 0 to para 5 . Majority of the parturients were of low socio-economic class. The parturients aged between 20 and 29 years were the highest group 131/250 (52.4\%) in the study followed by those aged between 30 and 39 years $65 / 250(26.0 \%)$. The least group was of those aged $40-49$ years 22/250 (8.8\%) [Table 1].

The highest prevalence of asymptomatic malaria parasitemia $(28 / 3287.5 \%)$ was found in the parturients who were $\leq 19$ years while the lowest prevalence of $(15 / 2268.2 \%)$ occurred in those who were 40-49 years but the association between age of paturients and asymptomatic malaria parastemia was not statistically significant $\left(\chi^{2}=3.713, P=0.32\right)$. Ninety-three (86.9\%) of the 107 nulliparous parturients had asymptomatic malaria parasitemia, while $69.4 \%$ (77/111) of the parturients of para 14 had asymptomatic malaria parasitemia. Twenty-four (75.0\%) of the grandmultiparous parturients had parasitemia. There was statistically significant association between parity and asymptomatic malaria parasitemia $\left(\chi^{2}=10.2, P=0.01\right)$. Majority of the parturients of social class $5(106 / 123(86.2 \%))$ were parasitemic. A significant percentage of paturients of social class $4(24 / 34(70.6 \%))$, social class $3(55 / 79(69.6 \%))$, social class $1(8 / 12(66.7 \%))$ and half of social class 2 had asymptomatic malaria parasitemia [Table 2]. There was a strong association between social class and asymptomatic malaria parasitemia.

Among the parasitemic parturients, $176 / 194$ (89.8\%) had one plus $(+)$ of parasite density, 18/194 (10.2\%) had parasite density of two plusses $(++)$. No parturient had a parasite density of three plusses $(+++)$ [Figure 1]. The only identified species of Plasmodium in the study was Plasmodium falciparum.

\section{DISCUSSION}

It was found that $194(77.6 \%)$ of the parturients had asymptomatic malaria parasitemia. Previous studies from different parts of Nigeria reported prevalence rates of 16-95.4\% among booked and unbooked pregnant women. ${ }^{[6-13]}$ In Benin and Abakaliki the prevalence was $20 \%$ and $16 \%$ as reported by Okpere et al. and Uneke et al. respectively, in their research among booked antenatal women. ${ }^{[6,13]}$ Higher prevalence of $58.4 \%, 63.8 \%, 89 \%$, and 95.4\% were reported in Enugu by Nwagha et al. Kano by Gajida et al. Ibadan by Falade et al. and Calabar by Agan et al. respectively, among unbooked pregnant women who came for antenatal booking. ${ }^{[7,8,11,12]}$ The prevalence of $77.6 \%$ in this study correlated with the findings among the unbooked pregnant women as shown above, even though it is lower than that in findings in Ibadan southwest Nigeria and Calabar south-south Nigeria and higher than that in findings

\begin{tabular}{lcc}
\hline \multicolumn{3}{c}{ Table 1: Socio-demographic characteristics of the parturients } \\
\hline Variables & Total number $(250)$ & $\%$ \\
\hline Age (years) & 32 & \\
$\leq 19$ & 131 & 12.8 \\
$20-29$ & 65 & 52.4 \\
$30-39$ & 22 & 26.0 \\
$40-49$ & - & 8.8 \\
$\geq 50$ & & - \\
Parity & 107 & \\
0 & 111 & 42.8 \\
$1-4$ & 32 & 44.4 \\
25 & & 12.8 \\
Social class & 12 & 4.8 \\
1 & 2 & 0.8 \\
2 & 79 & 31.6 \\
3 & 34 & 13.6 \\
4 & 123 & 49.2 \\
5
\end{tabular}

\begin{tabular}{|c|c|c|c|c|c|c|}
\hline \multicolumn{7}{|c|}{$\begin{array}{c}\text { Table 2: Socio-demographic characteristics and } \\
\text { asymptomatic malaria parasitemia }\end{array}$} \\
\hline \multirow[t]{2}{*}{ Variables } & \multicolumn{2}{|c|}{ Parasitemic } & \multicolumn{2}{|c|}{ Non-parasitemic } & \multirow[t]{2}{*}{$x^{2}$} & \multirow[t]{2}{*}{$P$ value } \\
\hline & Number & $\%$ & Number & $\%$ & & \\
\hline \multicolumn{7}{|c|}{ Age (years) } \\
\hline$\leq 19$ & 28 & 87.5 & 4 & 12.5 & 3.713 & 0.3 \\
\hline $20-29$ & 103 & 78.6 & 28 & 21.4 & & \\
\hline $20-39$ & 48 & 73.8 & 17 & 26.2 & & \\
\hline $40-49$ & 15 & 68.2 & 7 & 31.8 & & \\
\hline$\geq 50$ & - & - & - & - & & \\
\hline \multicolumn{7}{|l|}{ Parity } \\
\hline 0 & 93 & 86.9 & 14 & 13.1 & 10.02 & 0.01 \\
\hline $1-4$ & 77 & 69.4 & 34 & 30.6 & & \\
\hline$\geq 5$ & 24 & 75.0 & 8 & 25.0 & & \\
\hline \multicolumn{7}{|c|}{ Social class } \\
\hline 1 & 8 & 66.7 & 4 & 33.3 & 10.88 & 0.02 \\
\hline 2 & 1 & 50.0 & 1 & 50.0 & & \\
\hline 3 & 55 & 69.6 & 24 & 30.4 & & \\
\hline 4 & 24 & 70.6 & 10 & 29.4 & & \\
\hline 5 & 106 & 86.2 & 17 & 13.8 & & \\
\hline
\end{tabular}

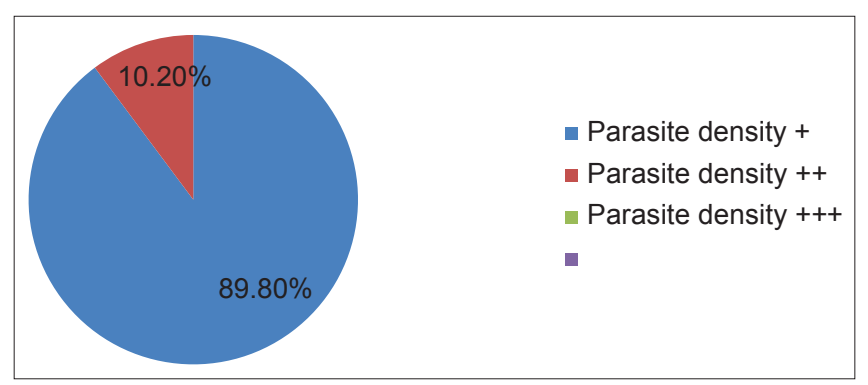

Figure 1: Parasite density of unbooked paturients with asymptomatic malaria parasitemia $\left({ }^{+}=1-10\right.$ parasites $/ 100$ thick film fields, ${ }^{++}=11-100$ parasites/100 thick film fields)

in Enugu southeast Nigeria and Kano northern Nigeria. The large variation in the reported prevalence of malaria parasitemia may be multifactorial. In an earlier study at Abakaliki a low prevalence rate of $16 \%$ was reported among booked women in the third trimester when interventions may have been undertaken. ${ }^{[13]}$ The studies that reported very high prevalence targeted women at booking visit when intervention was unlikely. ${ }^{[9,12]}$ The method of diagnosis is another factor that may explain the large variation in the reported prevalence of asymptomatic malaria parasitemia. 
Blood films are expected to be made without delay since morphological alteration of parasites occurs with storage of ethylenediametetra acetic acid (EDTA)-anticoagulated blood and it is advocated that the middle finger should be used for needle prick for blood smear collection as this improves identification of parasites. Different staining media ranging from Giemsa stain, Leishman stain, Field's stain, May-Grünwald-Giemsa (MGG), modified Field's stain could be used for the blood film and this may affect the level of diagnosis. There may also be error due to parallax and variations in individual visualization as well as identification ability. Other factors may include the study design, the targeted population, and the intensity of transmission.

Malaria parasitemia in pregnancy has been shown to be associated with younger maternal age with maternal age less than 20 years reported as a significant risk factor in Nigeria. ${ }^{17]}$ The association between maternal age and asymptomatic malaria parasitemia in this study was however not statistically significant though the greater percentage of the parturients of low age were parasitemic. This finding is at variance with other findings where maternal age less than 20 years was reported as a significant risk factor.

Asymptomatic malaria parasitemia was found to be associated with low parity in this study and majority of the parturients with parasitemia were primigravida. This has been demonstrated in previous studies. ${ }^{\mid 18,19]}$ This may be due to acquisition of parityspecific immunity which reduces susceptibility to malaria infestation during pregnancy as parity increases. ${ }^{[19]}$ Asymptomatic malaria parasitemia was also associated with low social class. Pregnant women of lowest social class (class 5) in this study had the highest malaria parasitemia with its occurrence being more than half (54.6\%) of all parturients with parasitemia in this study. This may be because these groups of parturients are more likely to live in a dirty environment where mosquito breed more, thus are more likely to get infested. Most of them are farmers, living in the rural setting with bushes around and may also not use preventive measures like insecticide treated nets.

\section{CONCLUSION}

The study demonstrated high prevalence of asymptomatic malaria parasitemia in labor among unbooked parturients. Asymptomatic malaria parasitemia is common among unbooked pregnant teenagers, primigravida, and those of low social class.

\section{Recommendation}

Health education of women within reproductive age on the importance of preconception and antenatal care as well as the need to scale up preventive measures against malaria in pregnancy is advocated.

A larger and community-based study is required in the future which will be more representative of the population.

\section{Limitations}

The study population was conducted in a tertiary hospital located in an urban area; thus, this may not be a true representation of the whole population within the state.

The semiquantitative method of parasite density estimation was used instead of calculating the absolute parasite density.

Congenital malaria parasite was not tested for in this study.

\section{ACKNOWLEDGMENTS}

We sincerely appreciate the management and laboratory staff of the Federal Teaching Hospital Abakaliki, Ebonyi State, Nigeria for their support in carrying out the laboratory investigations in this study.

We also wish to thank the research assistants and those who assisted us in the secretariat work as well data analysis.

\section{REFERENCES}

1. Opare-Addo HS, Odoi AT. Malaria in pregnancy. In: Kwawukume EY, Emuveyan EE, editors. Comprehensive Obstetrics in the Tropics. Accra Asante and Hittscher Printing Press Limited 2002; p. 25060.

2. Cox FE. History of human parasitology. Clin Microbiol Rev 2002; 15: 595-612.

3. Federal Ministry of Health. National Malaria Control Program in Nigeria. Roll Back Malaria Annual Report. Abuja: Malaria Control Program, Federal Ministry Health, 2005; p. 8-9.

4. Federal Ministry of Health. National malaria and vector control programme, training manual for management of malaria in Nigeria. Kaduna, Primo Wonder Limited, 2008 p. 13.

5. World Health Organization. Available from: http:/Malaria: High risk groups/pregnancy. [Last accessed 2011 Aug. 15]

6. Okpere EE. Malaria in pregnancy. In: Okpere EE, editor. Clinical Obstetrics. Revised ed. Benin UNIBEN Press 2004; p. 56-61.

7. Nwagha UI, Ugwu VO, Nwagha TU, Anyaehie BU. Asymptomatic plasmodium parasitaemia in pregnant Nigerian women: Almost a decade after Roll Back Malaria. Trans R Soc Trop Med Hyg 2009;103:16-20.

8. Agan T,EkabuaJ,Udoh AE, Ekanem EI, EfiokEE, Mgbekem M. Prevalence of anemia in women with asymptomatic malaria parasitemia at first antenatal visit at the University of Calabar Teaching Hospital, Calabar, Nigeria Int J Womens Health 2010; 2: 229-33.

9. Agan TU, Ekabua JE, Iklaki CU, Ibanga I. Prevalence of asymptomatic malaria parasitemia at first antenatal visit in UCTH Calabar. Asian Pac J Trop Med 2010; 1-5.

10. Anorlu RI, Odum CU, Essien EE. Asymptomatic malaria parasitaemia in pregnant women at booking in a primary health care facility in a periurban community in Lagos, Nigeria. Afr J Med Med Sci 
2001;30(suppl): 39-41.

11. Falade CO, Olayemi O, Dada-Adegbola HO, Aimakhu CO, Ademowo OG, Salako LA. Prevalence of malaria at booking among antenatal clients in a secondary health care facility in Ibadan, Nigeria. Afr J Reprod Health 2008;12: 142-52.

12. Gajida AU, Iliyasu Z, Zoakah AI. Malaria among antenatal clients attending primary health care facilities in Kano state, Nigeria. Ann Afr Med 2010; 9: 188-93.

13. Uneke CJ, Sunday-Adeoye I, Iyare FE, Ugwuja EI, Duhlinska DD. Impact of maternal plasmodium falciparum malaria and haematological parameters on pregnancy and its outcome in Southeastern Nigeria. J Vector Borne Dis 2007; 44: 285-90.

14. Umeora OU, Egwuatu VE. The role of unorthodox and traditional birth care in maternal mortality. Trop Doct 2010; 40: 13-7.

15. Daniel WW. Biostatistics: A Foundation for Analysis in the Health
Sciences. $7^{\text {th }}$ ed. New York: John Wiley and Sons; 1999; p. 9.

16. Lwanga SK, Lemeshow S. Sample Size Determination in Health Studies: A Practical Manual. Geneva: World Health Organization; 199;. p. 1, 25.

17. Mokuolu OA, Falade CO, Orogade AA, Okafor HU, Adedoyin OT, Oguonu TA, et al. Malaria at parturition in Nigeria: Current status and delivery outcome. Infect Dis Obstet Gynecol 2009;2009:473971

18. Brabin BJ, Romagosa C, Abdelgalil S, Menéndez C, Verhoeff FH, McGready R, et al. The sick placenta-the role of malaria. Placenta 2004; 25: 359-78.

19. Nosten F, McGready R, Mutabingwa T. Case management of malaria in pregnancy. Lancet Infect Dis 2007; 7: 118-25.

How to cite this article: ${ }^{* *}$

Source of Support: Nil, Conflict of Interest: None declared 